\title{
The Intuitionistic Fuzzy Linguistic Cosine Similarity Measure and Its Application in Pattern Recognition
}

\author{
Donghai Liu $\mathbb{D}$, ${ }^{1,2}$ Xiaohong Chen, ${ }^{1,3}$ and Dan Peng ${ }^{2}$ \\ ${ }^{1}$ School of Business, Central South University, Changsha, Hunan 410075, China \\ ${ }^{2}$ Department of Mathematics, Hunan University of Science and Technology, Xiangtan, Hunan 411201, China \\ ${ }^{3}$ Hunan University of Commerce, Changsha, Hunan 410205, China
}

Correspondence should be addressed to Donghai Liu; donghailiu@126.com

Received 7 December 2017; Revised 6 January 2018; Accepted 21 January 2018; Published 22 April 2018

Academic Editor: László T. Kóczy

Copyright (C) 2018 Donghai Liu et al. This is an open access article distributed under the Creative Commons Attribution License, which permits unrestricted use, distribution, and reproduction in any medium, provided the original work is properly cited.

\begin{abstract}
We propose the cosine similarity measures for intuitionistic fuzzy linguistic sets (IFLSs) and interval-valued intuitionistic fuzzy linguistic sets (IVIFLSs), which are expressed by the linguistic scale function based on the cosine function. Then, the weighted cosine similarity measure and the ordered weighted cosine similarity measure for IFLSs and IVIFLSs are introduced by taking into account the importance of each element, and the properties of the cosine similarity measures are also given. The main advantage of the proposed cosine similarity measures is that the decision-makers can flexibly select the linguistic scale function depending on the actual semantic situation. Finally, we present the application of the cosine similarity measures for intuitionistic fuzzy linguistic term sets and interval-valued intuitionistic fuzzy linguistic term sets to pattern recognition and medical diagnosis, and the existing cosine similarity measures are compared with the proposed cosine similarity measures by the illustrative example.
\end{abstract}

\section{Introduction}

The fuzzy set was proposed by Zadeh [1] and has achieved a great success in various fields, which is considered to be an effective tool to solve the decision-making problems, pattern recognition, and fuzzy inference [2-4]. Since the fuzzy set was put forward, it was extended in different aspects. One of the generalizations of fuzzy set is intuitionistic fuzzy set (IFS), which was introduced by Atanassov [5]. A typical feature of IFS is that the membership relations are represented by the membership degree and nonmembership degree, respectively. However, due to the fuzziness and uncertainty in the multiple criteria decision-making problems, it is difficult to use the exact values to present qualitative evaluation. At this time, people often provide their opinions in linguistic term sets. In some practical decision-making problems, the decision-maker regards the linguistic information as the values of linguistic variables; that is to say, the values of the variables are not represented by numerical values but are represented by linguistic values, such as "good," "better," "fair," "slightly worse," and "poor." Up to now, many people have studied the problem of linguistic multiple criteria decisionmaking, Herrera and Verdegay [6] proposed the linguistic assessments in group decision-making (GDM) problem in 1993, then Herrera et al. [7] proposed a consensus model for group decision-making based on linguistic evaluations information, and Herrera et al. [8] considered several group decision-making processes using linguistic ordered weighted averaging (LOWA) operator. Later, Xu [9] proposed a group decision-making method based on the uncertain linguistic ordered weighted geometric (LOWG) operators and the induced uncertain LOWG operators. Furthermore, Xu [10] presented the linguistic hybrid aggregation (LHA) operator and applied it to group decision-making.

However, in some practical decision-making problems, the decision-makers may have some indeterminacy in their linguistic evaluation; they cannot express their preferences by using only membership degree of a linguistic term. Then Wang et al. [11] proposed the intuitionistic fuzzy linguistic aggregation operators and applied them to multicriteria group decision-making problems. For example, $\left\langle s_{3},(0.2,0.6)\right\rangle$ is an intuitionistic fuzzy linguistic number 
(IFLN), 0.2 is the membership degree of the linguistic term $s_{3}$, and 0.6 is the nonmembership degree of the linguistic term $s_{3}$. The intuitionistic fuzzy linguistic sets (IFLSs) have made great progress in describing linguistic information and to some extent it can be regarded as an innovative construct. The research on this field has been growing rapidly [12-15].

On the other hand, similarity measure is an important topic in the fuzzy set theory, and it is widely used in some fields [16-19], such as pattern recognition, medical diagnosis, and citation analysis. One of the important similarity measures is the cosine similarity measure, which is defined in vector space. Ye [20] proposed the cosine similarity measure and the weighted cosine similarity measure between IFSs. Zhou et al. [21] presented the intuitionistic fuzzy ordered weighted cosine similarity measure and applied it to the group decision-making problem about the choice of investment plan. Liu et al. [22] presented the interval-valued intuitionistic fuzzy ordered weighted cosine similarity (IVIFOWCS) measure and applied it to the investment decisionmaking. As far as we know, the study of cosine similarity measures of intuitionistic fuzzy set has not been discussed. In the following, we will propose the cosine similarity measures of IFLSs and IVIFLSs. The main characteristics of the cosine similarity measures that we can calculate are based on linguistic term set by the linguistic scale function. Linguistic scale function between IFLSs and IVIFLSs was introduced by Wang et al. [23], which was used to calculate the Hausdorff distance between hesitant fuzzy linguistic numbers (HFLNs); it can assign different semantic values to the linguistic terms under different circumstances and improve the flexibility of the proposed cosine similarity measures.

The rest of the paper is organized as follows. In Section 2, some basic concepts of LTSs, IFSs, IFLSs, and linguistic scale functions are briefly reviewed. In Section 3, we first introduce the cosine similarity measures between IFLSs and then discussed some related properties. Furthermore, the weighted cosine similarity measure between IFLSs, the ordered weighted cosine similarity measure between IFLSs, and the ordered weighted cosine similarity measure between IVIFLSs are analyzed. In Section 4, we give the application of the proposed cosine similarity measures between IFLSs and IVIFLSs on pattern recognition and medical diagnosis and then make comparison analysis with the existing cosine similarity measures. The conclusions are given in Section 5.

\section{Preliminaries}

In this section, we will review and discuss some related basic concepts, including linguistic term sets (LTSs), intuitionistic fuzzy sets (IFSs), intuitionistic fuzzy linguistic sets (IFLSs), linguistic scale functions, the ordered weighted averaging (OWA) operator, and the cosine similarity measure between fuzzy sets.

2.1. Linguistic Term Set. In some practical problems, the information expressed by the numerical values may bring inconvenience. At this time it is suitable to use linguistic term set to express information.
Definition 1 (Herrera and Verdegay [6]). Suppose that $S=$ $\left\{s_{\alpha} \mid \alpha=0,1, \ldots, \tau\right\}$ is a finite and totally ordered discrete term set, where $s_{\alpha}$ represents a possible value for a linguistic variable; it satisfies the following characteristics:

(1) $s_{\alpha}+s_{\beta}=s_{\alpha+\beta}$

(2) $\lambda s_{\alpha}=s_{\lambda \alpha}$

(3) $s_{\alpha}>s_{\beta}$ if $\alpha>\beta$.

For example, a set of seven terms $S$ could be given as follows:

$$
\begin{aligned}
S= & \left\{s_{0}=\text { very low, } s_{1}=\text { low, } s_{2}=\text { slightly low, } s_{3}\right. \\
& =\text { normal, } s_{4}=\text { slightly high, } s_{5}=\text { high, } s_{6} \\
& =\text { very high }\} .
\end{aligned}
$$

The discrete linguistic term $S$ cannot usually adapt to the aggregated results. In order to represent these results accurately, $\mathrm{Xu}$ [10] extended the discrete term set $S$ to the continuous term set $\bar{S}=\left\{s_{\alpha} \mid \alpha \in[0, t]\right\}$, where $t(t>\tau)$ is a sufficiently large positive integer.

\subsection{Intuitionistic Fuzzy Set}

Definition 2 (Atanassov [5]). Given a fixed set $X=\left\{x_{1}, x_{2}\right.$, $\left.\ldots, x_{n}\right\}$, then an intuitionistic fuzzy set $\xi$ in $X$ is defined as

$$
\xi=\left\{\left(x, \mu_{\xi}(x), \nu_{\xi}(x)\right) \mid x \in X\right\},
$$

where $\mu_{\xi}(x)\left(0 \leq \mu_{\xi}(x) \leq 1\right)$ and $\nu_{\xi}(x)\left(0 \leq \nu_{\xi}(x) \leq\right.$ 1) represent the membership degree and nonmembership degree of $x$ to $\xi$, respectively, and they satisfy the condition: $0 \leq \mu_{\xi}(x)+\nu_{\xi}(x) \leq 1$.

For all $x \in X$, if $\pi_{\xi}(x)=1-\mu_{\xi}(x)-\nu_{\xi}(x)$, then $\pi_{\xi}(x)$ is called the hesitancy degree of $x$ to $\xi$.

On the basis of intuitionistic fuzzy set and linguistic term set, Wang et al. [11] presented the following intuitionistic fuzzy linguistic term set.

\subsection{Intuitionistic Fuzzy Linguistic Term Set}

Definition 3 (Wang et al. [11]). Let $X=\left\{x_{1}, x_{2}, \ldots, x_{n}\right\}$ be a fixed set and $s_{\gamma(x)} \in \bar{S}$ an intuitionistic fuzzy linguistic term set $\gamma$ in $X$ is defined as

$$
\gamma=\left\{\left\langle\left(x, s_{\gamma(x)}\right), \mu_{\gamma}(x), \nu_{\gamma}(x)\right\rangle \mid x \in X\right\},
$$

where $\mu_{\gamma}(x)$ and $\nu_{\gamma}(x)$ are the membership function and nonmembership function of the element $x$ to $s_{\gamma(x)}$, respectively, and $0 \leq \mu_{\gamma}(x)+\nu_{\gamma}(x) \leq 1(x \in X)$.

For all $x \in X$, let $\pi_{\gamma}(x)=1-\mu_{\gamma}(x)-\nu_{\gamma}(x)$ be the hesitancy function, which means the degree of hesitancy of $x$ to $s_{\gamma(x)}$.

2.4. Linguistic Scale Functions. One of the advantages of linguistic term set is that it can express uncertain information flexibly in practical problems, but if we use the subscript 
of linguistic terms directly in the process of operations, it may lose this advantage. The most important thing is to find effective tools to transform linguistic terms to numerical values. As we all know, linguistic scale function (Wang et al. [23]) is a mapping from linguistic term set $s_{i}$ to the real value $\eta_{i}$. The linguistic scale function can assign different semantic values to the linguistic terms under different circumstances. In practice, the linguistic scale functions are very popular because they are very flexible and they can give more deterministic results based on different semantics.

Definition 4 (Wang et al. [23]). Let $S=\left\{s_{i} \mid i=0,1, \ldots, 2 \tau\right\}$ be a linguistic term. If $\eta_{i}$ is a numeric value between 0 and 1 , then the linguistic scale function $f$ can be defined as follows:

$$
f: s_{i} \longrightarrow \eta_{i} \quad(i=0,1, \ldots, 2 \tau)
$$

where $0 \leq \eta_{0}<\eta_{1}<\cdots<\eta_{2 \tau} \leq 1$. The linguistic scale function is strictly monotonously increasing function with respect to the subscript of $s_{i}$; in fact, the function value $\eta_{i}$ represents the semantics of the linguistic terms.

Now we introduce three kinds of linguistic scale functions as follows:

$$
\text { (1) } f_{1}\left(s_{i}\right)=\eta_{i}=\frac{i}{2 \tau} \quad(i=0,1, \ldots, 2 \tau)
$$

The evaluation scale of the linguistic information expressed by $f_{1}\left(s_{i}\right)$ is divided on average.

$$
\text { (2) } \begin{aligned}
& f_{2}\left(s_{i}\right)=\eta_{i} \\
= & \begin{cases}\frac{c^{\tau}-c^{t-i}}{2 c^{\tau}-2}, & i=0,1, \ldots, \tau ; \\
\frac{c^{\tau}+c^{i-\tau}-2}{2 c^{\tau}-2}, & i=\tau+1, \tau+2, \ldots, 2 \tau .\end{cases}
\end{aligned}
$$

For linguistic scale function $f_{2}\left(s_{i}\right)$, the absolute deviation between adjacent language sets will increase when we extend it from the middle of the given set of language to both ends.

$$
\text { (3) } \begin{aligned}
& f_{3}\left(s_{i}\right)=\eta_{i} \\
= & \begin{cases}\frac{\tau^{\alpha^{\prime}}-(\tau-i)^{\alpha^{\prime}}}{2 \tau^{\alpha^{\prime}}}, & i=0,1, \ldots, \tau ; \\
\frac{\tau^{\beta^{\prime}}+(i-\tau)^{\beta^{\prime}}}{2 \tau^{\beta^{\prime}}}, & i=\tau+1, \tau+2, \ldots, 2 \tau .\end{cases}
\end{aligned}
$$

For linguistic scale function $f_{3}\left(s_{i}\right)$, the absolute deviation between adjacent language sets will decrease when we extend it from the middle of the given linguistic term set to both ends.

The above linguistic scale functions can be developed to $f^{*}: \bar{S} \rightarrow R^{+}$(where $R^{+}$is a nonnegative real number), which is a continuous and strictly monotonically increasing function.

2.5. The OWA Operator. The ordered weighted averaging (OWA) operator (Yager [24]) is an aggregation operator that includes the minimum, the average, and the maximum as special cases, which is defined as follows.

Definition 5 (Yager [24]). An $n$ dimension OWA operator is a mapping OWA: $R^{n} \rightarrow R$ that has an associated weighting vector $\omega$ with $\sum_{j=1}^{n} \omega_{j}=1\left(0 \leq \omega_{j} \leq 1\right)$, such that

$$
\operatorname{OWA}\left(\gamma_{1}, \gamma_{2}, \ldots, \gamma_{n}\right)=\sum_{j=1}^{n} \omega_{j} \gamma_{j}^{\prime}
$$

where $\gamma_{j}^{\prime}$ is the $j$ th largest of the arguments $\gamma_{1}, \gamma_{2}, \ldots, \gamma_{n}$.

\subsection{Cosine Similarity Measure for Fuzzy Sets}

Definition 6 (Salton and Mcgill [25]). Let $X=\left\{x_{1}, x_{2}, \ldots\right.$, $\left.x_{n}\right\}$; assume $\xi=\left\{\left(x_{j}, \mu_{\xi}\left(x_{j}\right)\right) \mid x_{j} \in X\right\}$ and $\sigma=\left\{\left(x_{j}, \mu_{\sigma}\left(x_{j}\right)\right) \mid\right.$ $\left.x_{j} \in X\right\}$ are two fuzzy sets; the cosine similarity measure between fuzzy sets $\xi$ and $\sigma$ is defined as follows:

$$
C_{\mathrm{FS}}(\xi, \sigma)=\frac{\sum_{j=1}^{n} \mu_{\xi}\left(x_{j}\right) \mu_{\sigma}\left(x_{j}\right)}{\sqrt{\sum_{j=1}^{n} \mu_{\xi}^{2}\left(x_{j}\right)} \sqrt{\sum_{j=1}^{n} \mu_{\sigma}^{2}\left(x_{j}\right)}} .
$$

The cosine similarity measure between fuzzy sets $\xi$ and $\sigma$ satisfies the following properties:

(1) $0 \leq C_{\mathrm{FS}}(\xi, \sigma) \leq 1$;

(2) $C_{\mathrm{FS}}(\xi, \sigma)=C_{\mathrm{FS}}(\sigma, \xi)$;

(3) for $j=1,2, \ldots, n$ if $\xi=\sigma$, that is, $\mu_{\xi}\left(x_{j}\right)=\mu_{\sigma}\left(x_{j}\right)$, then $C_{\mathrm{FS}}(\xi, \sigma)=1$.

\section{Cosine Similarity Measures for Intuitionistic Fuzzy Linguistic Term Sets}

3.1. Cosine Similarity Measure for Intuitionistic Fuzzy Linguistic Term Sets. At first, we will present cosine similarity measure for intuitionistic fuzzy linguistic term sets, which includes not only the membership degree and nonmembership degree of the IFLSs but also the linguistic scale function $f^{*}$.

Definition 7. Let $\beta=\left\{\left\langle\left(x_{j}, s_{\beta\left(x_{j}\right)}\right), \mu_{\beta}\left(x_{j}\right), \nu_{\beta}\left(x_{j}\right)\right\rangle \mid x_{j} \in X\right\}$ and $\gamma=\left\{\left\langle\left(x_{j}, s_{\gamma\left(x_{j}\right)}\right), \mu_{\gamma}\left(x_{j}\right), \nu_{\gamma}\left(x_{j}\right)\right\rangle \mid x_{j} \in X\right\}$ be two IFLSs in $X=\left\{x_{1}, x_{2}, \ldots, x_{m}\right\}$, and let $f^{*}$ be a linguistic scale function. Then the cosine similarity measure for intuitionistic fuzzy linguistic term sets between $\beta$ and $\gamma$ can be defined as follows:

$$
C_{\mathrm{IFLS}}(\beta, \gamma)=\frac{\sum_{j=1}^{m}\left[f^{*}\left(s_{\beta\left(x_{j}\right)}\right) \mu_{\beta}\left(x_{j}\right) f^{*}\left(s_{\gamma\left(x_{j}\right)}\right) \mu_{\gamma}\left(x_{j}\right)+f^{*}\left(s_{\beta\left(x_{j}\right)}\right) v_{\beta}\left(x_{j}\right) f^{*}\left(s_{\gamma\left(x_{j}\right)}\right) v_{\gamma}\left(x_{j}\right)\right]}{K \bullet H},
$$


where

K $=\sqrt{\sum_{j=1}^{m}\left[\left(f^{*}\left(s_{\beta\left(x_{j}\right)}\right) \mu_{\beta}\left(x_{j}\right)\right)^{2}+\left(f^{*}\left(s_{\beta\left(x_{j}\right)}\right) v_{\beta}\left(x_{j}\right)\right)^{2}\right]}$

$H$

$$
=\sqrt{\sum_{j=1}^{m}\left[\left(f^{*}\left(s_{\gamma\left(x_{j}\right)}\right) \mu_{\gamma}\left(x_{j}\right)\right)^{2}+\left(f^{*}\left(s_{\gamma\left(x_{j}\right)}\right) \nu_{\gamma}\left(x_{j}\right)\right)^{2}\right]} .
$$

The cosine similarity measure between IFLSs $\beta$ and $\gamma$ satisfies the following properties:

(1) $0 \leq C_{\text {IFLS }}(\beta, \gamma) \leq 1$;
(2) $C_{\mathrm{IFLS}}(\beta, \gamma)=C_{\mathrm{IFLS}}(\gamma, \beta)$;

(3) for $j=1,2, \ldots, n$ if $\beta=\gamma$, that is, $s_{\beta\left(x_{j}\right)}=$ $s_{\gamma\left(x_{j}\right)}, \mu_{\beta}\left(x_{j}\right)=\mu_{\gamma}\left(x_{j}\right)$, and $\nu_{\beta}\left(x_{j}\right)=\nu_{\gamma}\left(x_{j}\right)$, then $C_{\text {IFLS }}(\beta, \gamma)=1$.

Proof. Properties (1), (2), and (3) are obvious; here we omit the proof of property.

If we consider the weight of different element $x_{j} \in X$, now we introduce the intuitionistic fuzzy linguistic weighted cosine similarity measure $C_{\text {IFLWS }}$, which can be defined as follows.

Definition 8. Let $\beta=\left\{\left\langle\left(x_{j}, s_{\beta\left(x_{j}\right)}\right), \mu_{\beta}\left(x_{j}\right), v_{\beta}\left(x_{j}\right)\right\rangle \mid x_{j} \in X\right\}$ and $\gamma=\left\{\left\langle\left(x_{j}, s_{\gamma\left(x_{j}\right)}\right), \mu_{\gamma}\left(x_{j}\right), v_{\gamma}\left(x_{j}\right)\right\rangle \mid x_{j} \in X\right\}$ be two IFLSs in $X=\left\{x_{1}, x_{2}, \ldots, x_{m}\right\}$, and let $f^{*}$ be a linguistic scale function. Then the weighted cosine similarity measure for intuitionistic fuzzy linguistic term sets between $\beta$ and $\gamma$ can be defined as follows:

$$
C_{\text {IFLWS }}(\beta, \gamma)=\frac{\sum_{j=1}^{m} \omega_{j}\left[f^{*}\left(s_{\beta\left(x_{j}\right)}\right) \mu_{\beta}\left(x_{j}\right) f^{*}\left(s_{\gamma\left(x_{j}\right)}\right) \mu_{\gamma}\left(x_{j}\right)+f^{*}\left(s_{\beta\left(x_{j}\right)}\right) v_{\beta}\left(x_{j}\right) f^{*}\left(s_{\gamma\left(x_{j}\right)}\right) v_{\gamma}\left(x_{j}\right)\right]}{K_{1} \cdot H_{1}},
$$

where

$K_{1}$

$$
=\sqrt{\sum_{j=1}^{m} \omega_{j}\left[\left(f^{*}\left(s_{\beta\left(x_{j}\right)}\right) \mu_{\beta}\left(x_{j}\right)\right)^{2}+\left(f^{*}\left(s_{\beta\left(x_{j}\right)}\right) v_{\beta}\left(x_{j}\right)\right)^{2}\right]},
$$

$H_{1}$

$$
=\sqrt{\sum_{j=1}^{m} \omega_{j}\left[\left(f^{*}\left(s_{\gamma\left(x_{j}\right)}\right) \mu_{\gamma}\left(x_{j}\right)\right)^{2}+\left(f^{*}\left(s_{\gamma\left(x_{j}\right)}\right) v_{\gamma}\left(x_{j}\right)\right)^{2}\right]},
$$

$\omega_{j}$ is the weight of $x_{j} \in X$, and $\sum_{j=1}^{m} \omega_{j}=1\left(0 \leq \omega_{j} \leq 1\right)$.

Remark 9. For all $j=1,2, \ldots, m$, if we take $\omega_{j}=1 / m$, then the weighted cosine similarity measure $C_{\text {IFLWS }}(\beta, \gamma)$ is reduced to the cosine similarity measure $C_{\mathrm{IFLS}}(\beta, \gamma)$.
Based on the idea of the OWA operator, we present the intuitionistic fuzzy ordered weighted cosine similarity measure $C_{\text {IFLOWS }}(\beta, \gamma)$ between intuitionistic fuzzy linguistic term sets $\beta$ and $\gamma$ as follows.

Definition 10. Let $\beta=\left\{\left\langle\left(x_{j}, s_{\beta\left(x_{j}\right)}\right), \mu_{\beta}\left(x_{j}\right), \nu_{\beta}\left(x_{j}\right)\right\rangle \mid x_{j} \in X\right\}$ and $\gamma=\left\{\left\langle\left(x_{j}, s_{\gamma\left(x_{j}\right)}\right), \mu_{\gamma}\left(x_{j}\right), v_{\gamma}\left(x_{j}\right)\right\rangle \mid x_{j} \in X\right\}$ be two IFLSs in $X=\left\{x_{1}, x_{2}, \ldots, x_{m}\right\}$, and let $f^{*}$ be a linguistic scale function. Then the ordered weighted cosine similarity measure for intuitionistic fuzzy linguistic term sets between $\beta$ and $\gamma$ can be defined as follows:

$$
\begin{aligned}
& C_{\text {IFLOWS }}(\beta, \gamma) \\
& =\frac{\sum_{j=1}^{m} \omega_{j}\left[f^{*}\left(s_{\beta\left(x_{\varphi(j)}\right)}\right) \mu_{\beta}\left(x_{\varphi(j)}\right) f^{*}\left(s_{\gamma\left(x_{\varphi(j)}\right)}\right) \mu_{\gamma}\left(x_{\varphi(j)}\right)+G\right]}{K_{2} \bullet H_{2}},
\end{aligned}
$$

where

$$
\begin{aligned}
& K_{2}=\sqrt{\sum_{j=1}^{m} \omega_{j}\left[\left(f^{*}\left(s_{\beta\left(x_{\varphi(j)}\right)}\right) \mu_{\beta}\left(x_{\varphi(j)}\right)\right)^{2}+\left(f^{*}\left(s_{\beta\left(x_{\varphi(j)}\right)}\right) v_{\beta}\left(x_{\varphi(j)}\right)\right)^{2}\right]}, \\
& H_{2}=\sqrt{\sum_{j=1}^{m} \omega_{j}\left[\left(f^{*}\left(s_{\gamma\left(x_{\varphi(j)}\right)}\right) \mu_{\gamma}\left(x_{\varphi(j)}\right)\right)^{2}+\left(f^{*}\left(s_{\gamma\left(x_{\varphi(j)}\right)}\right) v_{\gamma}\left(x_{\varphi(j)}\right)\right)^{2}\right]}, \\
& G=f^{*}\left(s_{\beta\left(x_{\varphi(j)}\right)}\right) v_{\beta}\left(x_{\varphi(j)}\right) \cdot f^{*}\left(s_{\gamma\left(x_{\varphi(j)}\right)}\right) v_{\gamma}\left(x_{\varphi(j)}\right),
\end{aligned}
$$


the associated weighting vector $\omega=\left(\omega_{1}, \omega_{2}, \ldots, \omega_{m}\right)$ with $\sum_{j=1}^{m} \omega_{j}=1\left(0 \leq \omega_{j} \leq 1\right)$, and $(\varphi(1), \varphi(2), \ldots, \varphi(m))$ is any permutation of $(1,2, \ldots, m)$, such that

$$
\begin{aligned}
& f^{*}\left(s_{\beta\left(x_{\varphi(j)}\right)}\right) \mu_{\beta}\left(x_{\varphi(j)}\right) f^{*}\left(s_{\gamma\left(x_{\varphi(j)}\right)}\right) \mu_{\gamma}\left(x_{\varphi(j)}\right) \\
& +f^{*}\left(s_{\beta\left(x_{\varphi(j)}\right)}\right) \nu_{\beta}\left(x_{\varphi(j)}\right) f^{*}\left(s_{\gamma\left(x_{\varphi(j)}\right)}\right) \nu_{\gamma}\left(x_{\varphi(j)}\right) \\
& \quad \geq f^{*}\left(s_{\beta\left(x_{\varphi(j+1)}\right)}\right) \mu_{\beta}\left(x_{\varphi(j+1)}\right) f^{*}\left(s_{\gamma\left(x_{\varphi(j+1)}\right)}\right) \\
& \cdot \mu_{\gamma}\left(x_{\varphi(j+1)}\right)+f^{*}\left(s_{\beta\left(x_{\varphi(j+1)}\right)}\right) \nu_{\beta}\left(x_{\varphi(j+1)}\right) \\
& \cdot f^{*}\left(s_{\gamma\left(x_{\varphi(j+1)}\right)}\right) \nu_{\gamma}\left(x_{\varphi(j+1)}\right) .
\end{aligned}
$$

$C_{\text {IFLOWS }}$ is a similarity measure that uses the cosine similarity measure for IFLS in the OWA operator and the linguistic scale function is also applied.

Example 1. Let $S=\left\{s_{0}, s_{1}, s_{2}, s_{3}, s_{4}, s_{5}, s_{6}\right\}$ be the linguistic term set, and assume two intuitionistic fuzzy linguistic term sets are $\beta=\left\{\left\langle\left(x_{1}, s_{4}\right), 1,0\right\rangle,\left\langle\left(x_{2}, s_{5}\right), 0.8,0\right\rangle,\left\langle\left(x_{3}, s_{3}\right)\right.\right.$, $0.7,0.1\rangle\}, \gamma=\left\{\left\langle\left(x_{1}, s_{5}\right), 0.8,0.1\right\rangle,\left\langle\left(x_{2}, s_{5}\right), 1,0\right\rangle,\left\langle\left(x_{3}, s_{4}\right), 0.8\right.\right.$, $0.1\rangle\}$. If the linguistic scale function $f^{*}\left(s_{i}\right)=i / 2 \tau(\tau=3)$, by (10), we can get

$$
C_{\text {IFLS }}(\beta, \gamma)=0.9867
$$

If $\omega=\left(\omega_{1}, \omega_{2}, \omega_{3}\right)=(0.22,0.4,0.38)$, then the weighted cosine similarity measure $C_{\text {IFLWS }}(\beta, \gamma)=0.9889$.

If $\omega=\left(\omega_{1}, \omega_{2}, \omega_{3}\right)=(0.22,0.4,0.38)$, the ordered weighted cosine similarity measure $C_{\text {IFLoWS }}(\beta, \gamma)=0.9837$.
3.2. Cosine Similarity Measure for Interval-Valued Intuitionistic Fuzzy Linguistic Term Sets. In the intuitionistic fuzzy linguistic set $\gamma=\left\{\left\langle\left(x, s_{\gamma(x)}\right), \mu_{\gamma}(x), \nu_{\gamma}(x)\right\rangle \mid x \in X\right\}$, $\mu_{\gamma}(x)$ represents the membership degree of the element $x$ to $s_{\gamma(x)}$, and $v_{\gamma}(x)$ represents the nonmembership degree of the element $x$ to $s_{\gamma(x)}$; they are all precise values in $[0,1]$. But in some circumstances, it is difficult to provide the precise membership degree and nonmembership degree of the element $x$ to $s_{\gamma(x)}$. Atanassov and Gargov [26, 27] proposed the interval-valued intuitionistic fuzzy linguistic term set (IVIFLS), and the definition of interval-valued intuitionistic fuzzy linguistic term set is given as follows.

Definition 11. Let $X=\left\{x_{1}, x_{2}, \ldots, x_{n}\right\}$ be a fixed set and $s_{\widetilde{\gamma}(x)} \in$ $\bar{S}$ an interval-valued intuitionistic fuzzy linguistic term set $\tilde{\gamma}$ in $X$ is defined as

$$
\tilde{\gamma}=\left\{\left\langle\left(x, s_{\tilde{\gamma}(x)}\right), \mu_{\tilde{\gamma}}(x), \nu_{\widetilde{\gamma}}(x)\right\rangle \mid x \in X\right\},
$$

where $\mu_{\tilde{\gamma}}(x)=\left[\mu_{\tilde{\gamma} l}(x), \mu_{\tilde{\gamma} r}(x)\right] \subset[0,1]$ and $\nu_{\widetilde{\gamma}}(x)=$ $\left[\nu_{\tilde{\gamma} l}(x), \nu_{\tilde{\gamma} r}(x)\right] \subset[0,1]$ are the interval membership degree and the interval nonmembership degree of the element $x$ to $s_{\widetilde{\gamma}(x)}$, respectively, and $0 \leq \mu_{\tilde{\gamma} r}(x)+\nu_{\tilde{\gamma} r}(x) \leq 1(x \in X)$.

Based on the cosine similarity measure for intuitionistic fuzzy linguistic term sets $\beta$ and $\gamma$, we present the cosine similarity measure for interval-valued intuitionistic fuzzy linguistic term sets $\widetilde{\beta}$ and $\tilde{\gamma}$ as follows.

Definition 12. Let $\widetilde{\beta}=\left\{\left\langle\left(x_{j}, s_{\widetilde{\beta}\left(x_{j}\right)}\right), \mu_{\widetilde{\beta}}\left(x_{j}\right), \nu_{\widetilde{\beta}}\left(x_{j}\right)\right\rangle \mid x_{j} \in\right.$ $X\}$ and $\tilde{\gamma}=\left\{\left\langle\left(x_{j}, s_{\tilde{\gamma}\left(x_{j}\right)}\right), \mu_{\tilde{\gamma}}\left(x_{j}\right), \nu_{\tilde{\gamma}}\left(x_{j}\right)\right\rangle \mid x_{j} \in X\right\}$ be two IVIFLSs in $X=\left\{x_{1}, x_{2}, \ldots, x_{m}\right\}$, and let $f^{*}$ be a linguistic scale function. Then the cosine similarity measure for IVIFLSs between $\widetilde{\beta}$ and $\widetilde{\gamma}$ can be defined as follows:

$$
C_{\text {IVIFLS }}(\widetilde{\beta}, \tilde{\gamma})=\frac{\sum_{j=1}^{m}\left[f^{*}\left(s_{\tilde{\beta}\left(x_{j}\right)}\right) \mu_{\tilde{\beta} l}\left(x_{j}\right) f^{*}\left(s_{\tilde{\gamma}\left(x_{j}\right)}\right) \mu_{\tilde{\gamma} l}\left(x_{j}\right)+f^{*}\left(s_{\tilde{\beta}\left(x_{j}\right)}\right) \nu_{\tilde{\beta} l}\left(x_{j}\right) f^{*}\left(s_{\tilde{\gamma}\left(x_{j}\right)}\right) \nu_{\tilde{\gamma} l}\left(x_{j}\right)+U\right]}{Q \cdot R}
$$

where

$$
\begin{aligned}
& U=f^{*}\left(s_{\widetilde{\beta}\left(x_{j}\right)}\right) \mu_{\widetilde{\beta} r}\left(x_{j}\right) f^{*}\left(s_{\widetilde{\gamma}\left(x_{j}\right)}\right) \mu_{\tilde{\gamma} r}\left(x_{j}\right)+f^{*}\left(s_{\widetilde{\beta}\left(x_{j}\right)}\right) \nu_{\widetilde{\beta} r}\left(x_{j}\right) f^{*}\left(s_{\tilde{\gamma}\left(x_{j}\right)}\right) \nu_{\tilde{\gamma} r}\left(x_{j}\right), \\
& Q=\sqrt{\sum_{j=1}^{m}\left[\left(f^{*}\left(s_{\widetilde{\beta}\left(x_{j}\right)}\right) \mu_{\widetilde{\beta} l}\left(x_{j}\right)\right)^{2}+\left(f^{*}\left(s_{\widetilde{\beta}\left(x_{j}\right)}\right) \mu_{\widetilde{\beta} r}\left(x_{j}\right)\right)^{2}+\left(f^{*}\left(s_{\widetilde{\beta}\left(x_{j}\right)}\right) \nu_{\widetilde{\beta} l}\left(x_{j}\right)\right)^{2}+\left(f^{*}\left(s_{\widetilde{\beta}\left(x_{j}\right)}\right) \nu_{\widetilde{\beta} r}\left(x_{j}\right)\right)^{2}\right]}, \\
& R=\sqrt{\sum_{j=1}^{m}\left[\left(f^{*}\left(s_{\tilde{\gamma}\left(x_{j}\right)}\right) \mu_{\tilde{\gamma} l}\left(x_{j}\right)\right)^{2}+\left(f^{*}\left(s_{\tilde{\gamma}\left(x_{j}\right)}\right) \mu_{\tilde{\gamma} r}\left(x_{j}\right)\right)^{2}+\left(f^{*}\left(s_{\tilde{\gamma}\left(x_{j}\right)}\right) \nu_{\tilde{\gamma} l}\left(x_{j}\right)\right)^{2}+\left(f^{*}\left(s_{\tilde{\gamma}\left(x_{j}\right)}\right) \nu_{\tilde{\gamma} r}\left(x_{j}\right)\right)^{2}\right]} .
\end{aligned}
$$


The cosine similarity measure between IVIFLSs $\widetilde{\beta}$ and $\tilde{\gamma}$ also satisfies the following properties:

(1) $0 \leq C_{\operatorname{IVIFLS}}(\widetilde{\beta}, \widetilde{\gamma}) \leq 1$;

(2) $C_{\text {IVIFLS }}(\widetilde{\beta}, \widetilde{\gamma})=C_{\text {IVIFLS }}(\widetilde{\gamma}, \widetilde{\beta})$;

(3) for $j=1,2, \ldots, n$ if $\widetilde{\beta}=\tilde{\gamma}$, that is, $s_{\widetilde{\beta}\left(x_{j}\right)}=$ $s_{\widetilde{\gamma}\left(x_{j}\right)}, \mu_{\widetilde{\beta} l}\left(x_{j}\right)=\mu_{\tilde{\gamma} l}\left(x_{j}\right), \mu_{\tilde{\beta} r}\left(x_{j}\right)=\mu_{\widetilde{\gamma} r}\left(x_{j}\right), \nu_{\widetilde{\beta} l}\left(x_{j}\right)=$ $\nu_{\tilde{\gamma} l}\left(x_{j}\right)$ and $\nu_{\tilde{\beta} r}\left(x_{j}\right)=\nu_{\tilde{\gamma} r}\left(x_{j}\right)$, then $C_{\text {IVIFLS }}(\tilde{\beta}, \tilde{\gamma})=1$.
Next we go on studying the weighted cosine similarity measure between IVIFLSs; it can be defined as follows.

Definition 13. Let $\widetilde{\beta}=\left\{\left\langle\left(x_{j}, s_{\widetilde{\beta}\left(x_{j}\right)}\right), \mu_{\widetilde{\beta}}\left(x_{j}\right), \nu_{\widetilde{\beta}}\left(x_{j}\right)\right\rangle \mid x_{j} \in\right.$ $X\}$ and $\tilde{\gamma}=\left\{\left\langle\left(x_{j}, s_{\tilde{\gamma}\left(x_{j}\right)}\right), \mu_{\tilde{\gamma}}\left(x_{j}\right), \nu_{\tilde{\gamma}}\left(x_{j}\right)\right\rangle \mid x_{j} \in X\right\}$ be two IVIFLSs in $X=\left\{x_{1}, x_{2}, \ldots, x_{m}\right\}, \omega_{j}$ is the weight of $x_{j} \in X$, and $\sum_{j=1}^{m} \omega_{j}=1\left(0 \leq \omega_{j} \leq 1\right)$. Assume $f^{*}$ be a linguistic scale function, then the weighted cosine similarity measure between IVIFLSs $\widetilde{\beta}$ and $\tilde{\gamma}$ can be defined as follows:

$$
C_{\text {IVIFLWS }}(\widetilde{\beta}, \widetilde{\gamma})=\frac{\sum_{j=1}^{m} \omega_{j}\left[f^{*}\left(s_{\widetilde{\beta}\left(x_{j}\right)}\right) \mu_{\widetilde{\beta} l}\left(x_{j}\right) f^{*}\left(s_{\tilde{\gamma}\left(x_{j}\right)}\right) \mu_{\tilde{\gamma} l}\left(x_{j}\right)+f^{*}\left(s_{\widetilde{\beta}\left(x_{j}\right)}\right) v_{\widetilde{\beta} l}\left(x_{j}\right) f^{*}\left(s_{\widetilde{\gamma}\left(x_{j}\right)}\right) v_{\tilde{\gamma} l}\left(x_{j}\right)+U\right]}{Q_{1} \cdot R_{1}},
$$

where

$$
\begin{aligned}
U & =f^{*}\left(s_{\tilde{\beta}\left(x_{j}\right)}\right) \mu_{\tilde{\beta} r}\left(x_{j}\right) f^{*}\left(s_{\tilde{\gamma}\left(x_{j}\right)}\right) \mu_{\tilde{\gamma} r}\left(x_{j}\right)+f^{*}\left(s_{\tilde{\beta}\left(x_{j}\right)}\right) \nu_{\tilde{\beta} r}\left(x_{j}\right) f^{*}\left(s_{\tilde{\gamma}\left(x_{j}\right)}\right) \nu_{\tilde{\gamma} r}\left(x_{j}\right), \\
Q_{1} & =\sqrt{\sum_{j=1}^{m} \omega_{j}\left[\left(f^{*}\left(s_{\tilde{\beta}\left(x_{j}\right)}\right) \mu_{\widetilde{\beta} l}\left(x_{j}\right)\right)^{2}+\left(f^{*}\left(s_{\widetilde{\beta}\left(x_{j}\right)}\right) \mu_{\tilde{\beta} r}\left(x_{j}\right)\right)^{2}+\left(f^{*}\left(s_{\tilde{\beta}\left(x_{j}\right)}\right) \nu_{\widetilde{\beta} l}\left(x_{j}\right)\right)^{2}+\left(f^{*}\left(s_{\tilde{\beta}\left(x_{j}\right)}\right) \nu_{\widetilde{\beta} r}\left(x_{j}\right)\right)^{2}\right]}, \\
R_{1} & =\sqrt{\sum_{j=1}^{m} \omega_{j}\left[\left(f^{*}\left(s_{\tilde{\gamma}\left(x_{j}\right)}\right) \mu_{\tilde{\gamma} l}\left(x_{j}\right)\right)^{2}+\left(f^{*}\left(s_{\tilde{\gamma}\left(x_{j}\right)}\right) \mu_{\tilde{\gamma} r}\left(x_{j}\right)\right)^{2}+\left(f^{*}\left(s_{\tilde{\gamma}\left(x_{j}\right)}\right) \nu_{\tilde{\gamma} l}\left(x_{j}\right)\right)^{2}+\left(f^{*}\left(s_{\tilde{\gamma}\left(x_{j}\right)}\right) \nu_{\tilde{\gamma} r}\left(x_{j}\right)\right)^{2}\right] .}
\end{aligned}
$$

Remark 14. For all $j=1,2, \ldots, m$, if we take $\omega_{j}=1 / m$, then the weighted cosine similarity measure $C_{\text {IVIFLWS }}(\widetilde{\beta}, \widetilde{\gamma})$ is reduced to the cosine similarity measure $C_{\mathrm{IVIFLS}}(\widetilde{\beta}, \widetilde{\gamma})$.

Remark 15. For all $j=1,2, \ldots, m$, if $\mu_{\widetilde{\beta l}}\left(x_{j}\right)=$ $\mu_{\tilde{\beta} r}\left(x_{j}\right), \mu_{\tilde{\gamma} l}\left(x_{j}\right)=\mu_{\tilde{\gamma} r}\left(x_{j}\right)$, then the weighted cosine similarity measure $C_{\text {IVIFLWS }}(\widetilde{\beta}, \widetilde{\gamma})$ is reduced to the weighted cosine similarity measure $C_{\text {IFLWS }}(\beta, \gamma)$.

Remark 16. For all $j=1,2, \ldots, m$, if $\omega_{j}=1 / m$ and $\mu_{\widetilde{\beta} l}\left(x_{j}\right)=\mu_{\widetilde{\beta} r}\left(x_{j}\right), \mu_{\widetilde{\gamma} l}\left(x_{j}\right)=\mu_{\widetilde{\gamma} r}\left(x_{j}\right)$, then the weighted cosine similarity measure $C_{\text {IVIFLWS }}(\widetilde{\beta}, \widetilde{\gamma})$ is reduced to the cosine similarity measure $C_{\mathrm{IFLS}}(\beta, \gamma)$ for IFLSs.
Similarly, we also apply the OWA operator and the cosine similarity measure for IVIFLS to present the intervalvalued intuitionistic fuzzy ordered weighted cosine similarity measure $C_{\text {IVIFLOWS }}(\widetilde{\beta}, \widetilde{\gamma})$ between the interval-valued intuitionistic fuzzy linguistic term sets $\widetilde{\beta}$ and $\tilde{\gamma}$ as follows.

Definition 17. Let $\widetilde{\beta}=\left\{\left\langle\left(x_{j}, s_{\widetilde{\beta}\left(x_{j}\right)}\right), \mu_{\widetilde{\beta}}\left(x_{j}\right), v_{\widetilde{\beta}}\left(x_{j}\right)\right\rangle \mid x_{j} \in\right.$ $X\}$ and $\tilde{\gamma}=\left\{\left\langle\left(x_{j}, s_{\tilde{\gamma}\left(x_{j}\right)}\right), \mu_{\tilde{\gamma}}\left(x_{j}\right), \nu_{\tilde{\gamma}}\left(x_{j}\right)\right\rangle \mid x_{j} \in X\right\}$ be two IVIFLSs in $X=\left\{x_{1}, x_{2}, \ldots, x_{m}\right\}, \omega_{j}$ is the weight of $x_{j} \in X$, and $\sum_{j=1}^{m} \omega_{j}=1\left(0 \leq \omega_{j} \leq 1\right)$. Assume $f^{*}$ be a linguistic scale function, then the ordered weighted cosine similarity measure between IVIFLSs $\widetilde{\beta}$ and $\tilde{\gamma}$ can be defined as follows:

$$
C_{\text {IVIFLOWS }}(\tilde{\beta}, \tilde{\gamma})=\frac{\sum_{j=1}^{m} \omega_{j}\left[f^{*}\left(s_{\tilde{\beta}\left(x_{\varphi(j)}\right)}\right) f^{*}\left(s_{\tilde{\gamma}\left(x_{\varphi(j)}\right)}\right)\left(\mu_{\tilde{\beta} l}\left(x_{\varphi(j)}\right) \mu_{\tilde{\gamma} l}\left(x_{\varphi(j)}\right)+\nu_{\tilde{\beta} l}\left(x_{\varphi(j)}\right) \nu_{\tilde{\gamma} l}\left(x_{\varphi(j)}\right)\right)+U_{2}\right]}{Q_{2} \cdot R_{2}},
$$

where

$$
U_{2}=f^{*}\left(s_{\widetilde{\beta}\left(x_{\varphi(j)}\right)}\right) \mu_{\tilde{\beta} r}\left(x_{\varphi(j)}\right) f^{*}\left(s_{\widetilde{\gamma}\left(x_{\varphi(j)}\right)}\right) \mu_{\tilde{\gamma} r}\left(x_{\varphi(j)}\right)+f^{*}\left(s_{\widetilde{\beta}\left(x_{\varphi(j)}\right)}\right) \nu_{\widetilde{\beta} r}\left(x_{\varphi(j)}\right) f^{*}\left(s_{\tilde{\gamma}\left(x_{\varphi(j)}\right)}\right) \nu_{\tilde{\gamma} r}\left(x_{\varphi(j)}\right),
$$




$$
\begin{aligned}
Q_{2} & =\sqrt{\sum_{j=1}^{m} \omega_{j}\left[\left(f^{*}\left(s_{\tilde{\beta}\left(x_{\varphi(j)}\right)}\right)^{2}\left(\left(\mu_{\tilde{\beta} l}\left(x_{\varphi(j)}\right)\right)^{2}+\mu_{\tilde{\beta} r}\left(x_{\varphi(j)}\right)\right)^{2}\right)+\left(f^{*}\left(s_{\tilde{\beta}\left(x_{\varphi(j)}\right)}\right)^{2}\left(\left(v_{\tilde{\beta} l}\left(x_{\varphi(j)}\right)\right)^{2}+v_{\tilde{\beta} r}\left(x_{\varphi(j)}\right)\right)^{2}\right)\right]}, \\
R_{2} & =\sqrt{\sum_{j=1}^{m} \omega_{j}\left[\left(f^{*}\left(s_{\tilde{\gamma}\left(x_{\varphi(j)}\right)}\right)^{2}\left(\left(\mu_{\tilde{\gamma} l}\left(x_{\varphi(j)}\right)\right)^{2}+\mu_{\tilde{\gamma} r}\left(x_{\varphi(j)}\right)\right)^{2}\right)+\left(f^{*}\left(s_{\tilde{\gamma}\left(x_{\varphi(j)}\right)}\right)^{2}\left(\left(v_{\tilde{\gamma} l}\left(x_{\varphi(j)}\right)\right)^{2}+v_{\tilde{\gamma} r}\left(x_{\varphi(j)}\right)\right)^{2}\right)\right]},
\end{aligned}
$$

and $(\varphi(1), \varphi(2), \ldots, \varphi(m))$ is any permutation of $(1,2, \ldots, m)$, such that

$$
\begin{aligned}
& f^{*}\left(s_{\tilde{\beta}\left(x_{\varphi(j)}\right)}\right) f^{*}\left(s_{\tilde{\gamma}\left(x_{\varphi(j)}\right)}\right)\left[\mu_{\tilde{\beta} l}\left(x_{\varphi(j)}\right) \mu_{\tilde{\gamma} l}\left(x_{\varphi(j)}\right)\right. \\
& +v_{\widetilde{\beta} l}\left(x_{\varphi(j)}\right) v_{\widetilde{\gamma} l}\left(x_{\varphi(j)}\right)+\mu_{\tilde{\beta} r}\left(x_{\varphi(j)}\right) \mu_{\tilde{\gamma} r}\left(x_{\varphi(j)}\right) \\
& \left.+\nu_{\widetilde{\beta} r}\left(x_{\varphi(j)}\right) \nu_{\widetilde{\gamma} r}\left(x_{\varphi(j)}\right)\right] \geq f^{*}\left(s_{\widetilde{\beta}\left(x_{\varphi(j+1)}\right)}\right) \\
& \cdot f^{*}\left(s_{\tilde{\gamma}\left(x_{\varphi(j+1)}\right)}\right)\left[\mu_{\widetilde{\beta} l}\left(x_{\varphi(j+1)}\right) \mu_{\tilde{\gamma} l}\left(x_{\varphi(j+1)}\right)\right. \\
& +v_{\widetilde{\beta} l}\left(x_{\varphi(j+1)}\right) v_{\widetilde{\gamma} l}\left(x_{\varphi(j+1)}\right) \\
& +\mu_{\tilde{\beta} r}\left(x_{\varphi(j+1)}\right) \mu_{\tilde{\gamma} r}\left(x_{\varphi(j+1)}\right) \\
& \left.+v_{\tilde{\beta} r}\left(x_{\varphi(j+1)}\right) v_{\tilde{\gamma} r}\left(x_{\varphi(j+1)}\right)\right] \text {. }
\end{aligned}
$$

\section{Applications of the Cosine Similarity Measure}

In this section, we will apply the cosine similarity measures of IFLSs and IVIFLSs to pattern recognition and medical diagnosis.

\subsection{Intuitionistic Fuzzy Cosine Similarity Measure for Pattern \\ Recognition}

Example 2. Let $X=\left\{x_{1}, x_{2}, x_{3}, x_{4}\right\}$, the linguistic term set $S=\left\{s_{0}=\right.$ very poor, $s_{1}=$ poor, $s_{2}=$ slightly poor, $s_{3}=$ fair, $s_{4}=$ slightly good, $s_{5}=$ good, $s_{6}=$ very good $\}$. We consider some known patterns $Q_{1}, Q_{2}, Q_{3}, Q_{4}$, which are represented by the IFLSs as follows:

$$
\begin{gathered}
Q_{1}=\left\{\left\langle\left(x_{1}, s_{4}\right), 0.8,0.1\right\rangle,\left\langle\left(x_{2}, s_{3}\right), 1,0\right\rangle,\right. \\
\left.\left\langle\left(x_{3}, s_{5}\right), 0.7,0.1\right\rangle,\left\langle\left(x_{4}, s_{3}\right), 0.6,0.3\right\rangle\right\}, \\
Q_{2}=\left\{\left\langle\left(x_{1}, s_{3}\right), 0.9,0\right\rangle,\left\langle\left(x_{2}, s_{3}\right), 0.8,0.1\right\rangle,\right. \\
\left.\left\langle\left(x_{3}, s_{4}\right), 0.9,0.1\right\rangle,\left\langle\left(x_{4}, s_{3}\right), 0.6,0.3\right\rangle\right\}, \\
Q_{3}=\left\{\left\langle\left(x_{1}, s_{4}\right), 0.7,0.1\right\rangle,\left\langle\left(x_{2}, s_{4}\right), 0.9,0\right\rangle,\right. \\
\left.\left\langle\left(x_{3}, s_{5}\right), 0.8,0.1\right\rangle,\left\langle\left(x_{4}, s_{3}\right), 0.8,0.1\right\rangle\right\}, \\
Q_{4}=\left\{\left\langle\left(x_{1}, s_{5}\right), 0.6,0.2\right\rangle,\left\langle\left(x_{2}, s_{4}\right), 0.7,0.2\right\rangle,\right. \\
\left.\left\langle\left(x_{3}, s_{5}\right), 0.8,0.2\right\rangle,\left\langle\left(x_{4}, s_{2}\right), 0.7,0.2\right\rangle\right\} .
\end{gathered}
$$

If an unknown pattern $Q=\left\{\left\langle\left(x_{1}, s_{4}\right), 0.8,0.2\right\rangle,\left\langle\left(x_{2}, s_{4}\right)\right.\right.$, $\left.1,0\rangle,\left\langle\left(x_{3}, s_{4}\right), 0.8,0.1\right\rangle,\left\langle\left(x_{4}, s_{3}\right), 0.8,0.2\right\rangle\right\}$, in order to classify the pattern $Q$ in $Q_{1}, Q_{2}, Q_{3}, Q_{4}$, we can calculate the weighted cosine similarity measure between $Q$ and $Q_{i}(i=$ $1,2,3,4)$, respectively. The best $Q_{i}$ is derived by $R=$ $\arg \max _{1 \leq i \leq 4}\left\{C_{\text {IFLWS }}\left(Q_{i}, Q\right)\right\}$. Assume the weight of $\left(x_{1}, x_{2}\right.$, $\left.x_{3}, x_{4}\right)$ is $(0.22,0.3,0.2,0.28)$ and let the linguistic scale function $f^{*}=f_{1}\left(s_{i}\right)=i / 2 \tau(\tau=3)$, and by applying (10), (12), and (14), we obtain the cosine similarity measures between $Q$ and $Q_{i}(i=1,2,3,4)$, and the results are shown in Table 1.

From the result shown in Table 1, we can conclude that the pattern $Q$ belongs to the pattern $Q_{3}$.

To illustrate the influence of the linguistic scale function $f^{*}$ on decision-making, we utilize the different linguistic scaling functions in the proposed cosine similarity measures.

Let

$$
\begin{aligned}
f^{*} & =f_{2}\left(s_{i}\right) \\
& = \begin{cases}\frac{c^{\tau}-c^{\tau-i}}{2 c^{\tau}-2}, & i=0,1, \ldots, \tau, \\
\frac{c^{\tau}+c^{i-\tau}-2}{2 c^{\tau}-2}, & i=\tau+1, \tau+2, \ldots, 2 \tau,\end{cases}
\end{aligned}
$$

and $c=1.4, \tau=3$, then the results are listed in Table 2 .

If

$$
f_{3}^{*}\left(s_{i}\right)= \begin{cases}\frac{\tau^{\alpha^{\prime}}-(\tau-i)^{\alpha^{\prime}}}{2 \tau^{\alpha^{\prime}}}, & i=0,1, \ldots, \tau, \\ \frac{\tau^{\beta^{\prime}}+(i-\tau)^{\beta^{\prime}}}{2 \tau^{\beta^{\prime}}}, & i=\tau+1, \tau+2, \ldots, 2 \tau,\end{cases}
$$

and $\tau=3, \alpha^{\prime}=\beta^{\prime}=0.8$, the results of the cosine similarity measure are shown in Table 3 .

As we can see from Tables 2 and 3, we know that the pattern $Q$ should be classified in $Q_{3}$ in most cases. It is a little different when the linguistic scale function $f^{*}=f_{2}\left(s_{i}\right)$ (the linguistic scale function which can be considered as the actual semantic situation), then the decision-makers can select the appropriate linguistic scale function $f^{*}$ according to their interests.

4.2. Interval-Valued Intuitionistic Fuzzy Cosine Similarity Measure for Medical Diagnosis. In this subsection, we will utilize the interval-valued intuitionistic fuzzy cosine similarity measure to discuss the medical diagnosis. In fact, it is also a pattern recognition problem. 
TABLE 1: Intuitionistic fuzzy linguistic cosine similarity measures for $f^{*}=f_{1}\left(s_{i}\right)$.

\begin{tabular}{lcccc}
\hline & $Q_{1}$ & $Q_{2}$ & $Q_{3}$ & $Q_{4}$ \\
\hline$C_{\text {IFLS }}\left(Q, Q_{i}\right)$ & 0.9828 & 0.9589 & 0.9859 & 0.9476 \\
$C_{\text {IFLWS }}\left(Q, Q_{i}\right)$ & 0.9826 & 0.9587 & 0.9853 & 0.9488 \\
$C_{\text {IFLOWS }}\left(Q, Q_{i}\right)$ & 0.9824 & 0.9582 & 0.9849 & 0.9473 \\
\hline
\end{tabular}

TABLE 2: Intuitionistic fuzzy linguistic cosine similarity measures for $f^{*}=f_{2}\left(s_{i}\right)$.

\begin{tabular}{lcccc}
\hline & $Q_{1}$ & $Q_{2}$ & $Q_{3}$ & $Q_{4}$ \\
\hline$C_{\text {IFLS }}\left(Q, Q_{i}\right)$ & 0.9861 & 0.9655 & 0.9854 & 0.9600 \\
$C_{\text {IFLWS }}\left(Q, Q_{i}\right)$ & 0.9862 & 0.9654 & 0.9871 & 0.9598 \\
$C_{\text {IFLOWS }}\left(Q, Q_{i}\right)$ & 0.9853 & 0.9849 & 0.9848 & 0.9713 \\
\hline
\end{tabular}

TABLE 3: Intuitionistic fuzzy linguistic cosine similarity measures for $f^{*}=f_{3}\left(s_{i}\right)$.

\begin{tabular}{lcccc}
\hline & $Q_{1}$ & $Q_{2}$ & $Q_{3}$ & $Q_{4}$ \\
\hline$C_{\text {IFLS }}\left(Q, Q_{i}\right)$ & 0.9806 & 0.9530 & 0.9875 & 0.9494 \\
$C_{\text {IFLWS }}\left(Q, Q_{i}\right)$ & 0.9800 & 0.9526 & 0.9889 & 0.9472 \\
$C_{\text {IFLOWS }}\left(Q, Q_{i}\right)$ & 0.9805 & 0.9519 & 0.9870 & 0.9458 \\
\hline
\end{tabular}

Example 3. Let us consider that the doctor makes a diagnosis $D \quad=\quad\left\{D_{1}\right.$ (viral fever), $D_{2}$ (typhoid), $D_{3}$ (pneumonia), $D_{4}$ (stomach problem) $\}$, and assume that a set of symptoms $X=\left\{x_{1}\right.$ (fever), $x_{2}$ (cough), $x_{3}$ (headache), $x_{4}$ (stomach pain) $\}$, and each symptom can be presented as the linguistic term set $S_{1}=\left\{s_{0}=\right.$ verylow, $s_{1}=$ low, $s_{2}=$ slightly low,$s_{3}=$ normal, $s_{4}=$ slightly high,$s_{5}=$ high, $s_{6}=$ very high $\}, S_{j}=\left\{s_{0}=\right.$ none, $s_{1}=$ very slight, $s_{2}=$ slight, $s_{3}=$ a little terrible, $s_{4}=$ terrible, $s_{5}=$ very terrible, $s_{6}=$ insufferable $\}(j=2,3,4)$. Suppose that the patient $P$ has all the symptoms, which is represented by the IVIFLS as follows:

$$
\begin{gathered}
P(\text { patient })=\left\{\left\langle\left(x_{1}, s_{3}\right),[0.4,0.5],[0.2,0.3]\right\rangle,\right. \\
\left\langle\left(x_{2}, s_{4}\right),[0.7,0.8],[0.1,0.2]\right\rangle, \\
\left\langle\left(x_{3}, s_{3}\right),[0.9,1],[0,0.1]\right\rangle, \\
\left.\left\langle\left(x_{4}, s_{1}\right),[0.3,0.5],[0.2,0.4]\right\rangle\right\} .
\end{gathered}
$$

Each symptom diagnosis $D_{i}(i=1,2,3,4)$ can be also represented as IVIFLSs as follows:

$$
\begin{gathered}
D_{1}=\left\{\left\langle\left(x_{1}, s_{4}\right),[0.8,0.9],[0,0.1]\right\rangle,\right. \\
\left\langle\left(x_{2}, s_{3}\right),[0.7,0.9],[0.1,0.2]\right\rangle, \\
\left\langle\left(x_{3}, s_{4}\right),[0.5,0.6],[0.2,0.3]\right\rangle, \\
\left.\left\langle\left(x_{4}, s_{0}\right),[0.7,0.9],[0.1,0.2]\right\rangle\right\}, \\
D_{2}=\left\{\left\langle\left(x_{1}, s_{3}\right),[0.5,0.6],[0.1,0.3]\right\rangle,\right. \\
\left\langle\left(x_{2}, s_{4}\right),[0.8,0.9],[0,0.1]\right\rangle,
\end{gathered}
$$

TABLE 4: Interval intuitionistic fuzzy linguistic cosine similarity measures for $f^{*}=f_{1}\left(s_{i}\right)$.

\begin{tabular}{lcccc}
\hline & $D_{1}$ & $D_{2}$ & $D_{3}$ & $D_{4}$ \\
\hline$C_{\text {IVIFLS }}\left(P, D_{i}\right)$ & 0.8685 & 0.9004 & 0.7748 & 0.5481 \\
$C_{\text {IVIFLWS }}\left(P, D_{i}\right)$ & 0.8809 & 0.9119 & 0.8619 & 0.6102 \\
$C_{\text {IVIFLOWS }}\left(P, D_{i}\right)$ & 0.8967 & 0.9267 & 0.8212 & 0.5310 \\
\hline
\end{tabular}

TABLE 5: Interval intuitionistic fuzzy linguistic cosine similarity measures for $f^{*}=f_{2}\left(s_{i}\right)$.

\begin{tabular}{lcccc}
\hline & $D_{1}$ & $D_{2}$ & $D_{3}$ & $D_{4}$ \\
\hline$C_{\text {IVIFLS }}\left(P, D_{i}\right)$ & 0.8842 & 0.8966 & 0.7865 & 0.6839 \\
$C_{\text {IVIFLWS }}\left(P, D_{i}\right)$ & 0.9003 & 0.9139 & 0.8657 & 0.7354 \\
$C_{\text {IVIFLOWS }}\left(P, D_{i}\right)$ & 0.9051 & 0.9247 & 0.8292 & 0.6726 \\
\hline
\end{tabular}

TABLE 6: Interval intuitionistic fuzzy linguistic cosine similarity measures for $f^{*}=f_{3}\left(s_{i}\right)$.

\begin{tabular}{lcccc}
\hline & $D_{1}$ & $D_{2}$ & $D_{3}$ & $D_{4}$ \\
\hline$C_{\text {IVIFLS }}\left(P, D_{i}\right)$ & 0.8550 & 0.9044 & 0.7724 & 0.4681 \\
$C_{\text {IVIFLWS }}\left(P, D_{i}\right)$ & 0.8641 & 0.9247 & 0.8616 & 0.5297 \\
$C_{\text {IVIFLOWS }}\left(P, D_{i}\right)$ & 0.8880 & 0.9292 & 0.8181 & 0.4486 \\
\hline
\end{tabular}

$$
\begin{gathered}
\left\langle\left(x_{3}, s_{3}\right),[0.6,0.8],[0.1,0.2]\right\rangle, \\
\left.\left\langle\left(x_{4}, s_{2}\right),[0.4,0.6],[0.1,0.2]\right\rangle\right\}, \\
D_{3}=\left\{\left\langle\left(x_{1}, s_{5}\right),[0.7,0.8],[0.1,0.2]\right\rangle,\right. \\
\left\langle\left(x_{2}, s_{5}\right),[0.7,0.9],[0,0.1]\right\rangle, \\
\left\langle\left(x_{3}, s_{1}\right),[0.4,0.6],[0.2,0.4]\right\rangle, \\
\left.\left\langle\left(x_{4}, s_{1}\right),[0.3,0.5],[0.2,0.4]\right\rangle\right\}, \\
D_{4}=\left\{\left\langle\left(x_{1}, s_{1}\right),[0.8,0.9],[0,0.1]\right\rangle,\right. \\
\left\langle\left(x_{2}, s_{2}\right),[0.7,0.8],[0.1,0.2]\right\rangle, \\
\left\langle\left(x_{3}, s_{1}\right),[0.7,0.9],[0,0.1]\right\rangle, \\
\left.\left\langle\left(x_{4}, s_{5}\right),[0.8,0.9],[0,0.1]\right\rangle\right\}
\end{gathered}
$$

We assume that the weight vector of $\left(x_{1}, x_{2}, x_{3}, x_{4}\right)$ is $(0.25,0.4,0.15,0.2)$ and let the linguistic scale function $f^{*}=$ $f_{1}\left(s_{i}\right)=i / 2 \tau(\tau=3)$, and by applying (19), (21), and (23), we obtain the cosine similarity measures between $P$ and $D_{i}(i=$ $1,2,3,4)$, and the results are shown in Table 4.

From the results of Table 4, we can see that the diagnosis of the patient $P$ is $D_{2}$ (typhoid).

To illustrate the influence of the linguistic scale function $f^{*}$ on decision-making, we utilize the different linguistic scaling functions $f_{2}\left(s_{i}\right)$ and $f_{3}\left(s_{i}\right)$ (the parameters are the same as Example 2) in the cosine similarity measures between IVIFLSs, and the results are shown in Tables 5 and 6. 
TABLE 7: Cosine similarity measures for intuitionistic fuzzy sets.

\begin{tabular}{lcccc}
\hline & $Q_{1}^{\prime}$ & $Q_{2}^{\prime}$ & $Q_{3}^{\prime}$ & $Q_{4}^{\prime}$ \\
\hline$C_{\mathrm{IFS}}\left(Q^{\prime}, Q_{i}^{\prime}\right)$ & 0.9902 & 0.9733 & 0.9954 & 0.9772 \\
$C_{\mathrm{IFWS}}\left(Q^{\prime}, Q_{i}^{\prime}\right)$ & 0.9898 & 0.9734 & 0.9956 & 0.9761 \\
$C_{\mathrm{IFOWS}}\left(Q^{\prime}, Q_{i}^{\prime}\right)$ & 0.9890 & 0.9742 & 0.9949 & 0.9773 \\
\hline
\end{tabular}

In these cases, we still find that the diagnosis of the patient $P$ should be classified in $D_{2}$ (typhoid).

4.3. Comparison Analysis with Existing Cosine Similarity Measure. To illustrate the validity and advantage of the proposed cosine similarity measure for pattern recognition and medical diagnosis, we now use the existing cosine similarity measure between intuitionistic fuzzy sets and interval-valued intuitionistic fuzzy sets (Ye [20]) for comparison analysis. Here we consider the intuitionistic fuzzy set in Example 2 and interval-valued intuitionistic fuzzy set in Example 3, respectively.

Example $2^{\prime}$. Let $X=\left\{x_{1}, x_{2}, x_{3}, x_{4}\right\}$; we consider some known patterns $Q_{1}^{\prime}, Q_{2}^{\prime}, Q_{3}^{\prime}, Q_{4}^{\prime}$, which are represented by the IFSs as follows:

$$
\begin{aligned}
Q_{1}^{\prime} & =\left\{\left\langle x_{1}, 0.8,0.1\right\rangle,\left\langle x_{2}, 1,0\right\rangle,\left\langle x_{3}, 0.7,0.1\right\rangle,\right. \\
& \left.\left\langle x_{4}, 0.6,0.3\right\rangle\right\}, \\
Q_{2}^{\prime} & =\left\{\left\langle x_{1}, 0.9,0\right\rangle,\left\langle x_{2}, 0.8,0.1\right\rangle,\left\langle x_{3}, 0.9,0.1\right\rangle,\right. \\
& \left.\left\langle x_{4}, 0.6,0.3\right\rangle\right\}, \\
Q_{3}^{\prime} & =\left\{\left\langle x_{1}, 0.7,0.1\right\rangle,\left\langle x_{2}, 0.9,0\right\rangle,\left\langle x_{3}, 0.8,0.1\right\rangle,\right. \\
& \left.\left\langle x_{4}, 0.8,0.1\right\rangle\right\}, \\
Q_{4}^{\prime} & =\left\{\left\langle x_{1}, 0.6,0.2\right\rangle,\left\langle x_{2}, 0.7,0.2\right\rangle,\left\langle x_{3}, 0.8,0.2\right\rangle,\right. \\
& \left.\left\langle x_{4}, 0.7,0.2\right\rangle\right\} .
\end{aligned}
$$

The unknown pattern $Q^{\prime}=\left\{\left\langle x_{1}, 0.8,0.2\right\rangle,\left\langle x_{2}, 1,0\right\rangle,\left\langle x_{3}\right.\right.$, $\left.0.8,0.1\rangle,\left\langle x_{4}, 0.8,0.2\right\rangle\right\}$ and the weight of $\left(x_{1}, x_{2}, x_{3}, x_{4}\right)$ is still $(0.22,0.3,0.2,0.28)$. We can calculate the intuitionistic fuzzy cosine similarity measure $C_{\text {IFS }}$, the intuitionistic fuzzy weighted cosine similarity measure $C_{\mathrm{IFWS}}$, and the intuitionistic fuzzy ordered weighted cosine similarity measure $C_{\text {IFOws }}$ between $Q^{\prime}$ and $Q_{i}^{\prime}(i=1,2,3,4)$, respectively, and the results are shown in Table 7.

The results of Table 7 show that the pattern $Q^{\prime}$ can be classified in $Q_{3}^{\prime}$; this shows the effectiveness of the proposed cosine similarity measures in this paper.

Example $3^{\prime}$. Let us consider that the doctor makes a diagnosis $D=\left\{D_{1}^{\prime}\right.$ (viral fever), $D_{2}^{\prime}$ (typhoid), $D_{3}^{\prime}$ (pneumonia), $D_{4}^{\prime}$ (stomach problem) $\}$, and assume that a set of symptoms $X=\left\{x_{1}(\right.$ fever $), x_{2}$ (cough), $x_{3}$ (headache),
TABLE 8: Cosine similarity measures for interval-valued intuitionistic fuzzy sets.

\begin{tabular}{lcccc}
\hline & $D_{1}^{\prime}$ & $D_{2}^{\prime}$ & $D_{3}^{\prime}$ & $D_{4}^{\prime}$ \\
\hline$C_{\mathrm{IFS}}\left(P^{\prime}, D_{i}^{\prime}\right)$ & 0.9173 & 0.9617 & 0.8757 & 0.9546 \\
$C_{\mathrm{IFWS}}\left(P^{\prime}, D_{i}^{\prime}\right)$ & 0.9341 & 0.9638 & 0.9019 & 0.9566 \\
$C_{\mathrm{IFOWS}}\left(P^{\prime}, D_{i}^{\prime}\right)$ & 0.9423 & 0.9650 & 0.8752 & 0.9614 \\
\hline
\end{tabular}

$x_{4}$ (stomach pain)\}, and each symptom can be represented as the interval-valued intuitionistic fuzzy set as follows:

$$
\begin{aligned}
D_{1}^{\prime} & =\left\{\left\langle x_{1},[0.8,0.9],[0,0.1]\right\rangle,\right. \\
& \left\langle x_{2},[0.7,0.9],[0.1,0.2]\right\rangle,\left\langle x_{3},[0.5,0.6],[0.2,0.3]\right\rangle, \\
& \left.\left\langle x_{4},[0.7,0.9],[0.1,0.2]\right\rangle\right\} \\
D_{2}^{\prime} & =\left\{\left\langle x_{1},[0.5,0.6],[0.1,0.3]\right\rangle\right. \\
& \left\langle x_{2},[0.8,0.9],[0,0.1]\right\rangle,\left\langle x_{3},[0.6,0.8],[0.1,0.2]\right\rangle, \\
& \left.\left\langle x_{4},[0.4,0.6],[0.1,0.2]\right\rangle\right\} \\
D_{3}^{\prime} & =\left\{\left\langle x_{1},[0.7,0.8],[0.1,0.2]\right\rangle\right. \\
& \left\langle x_{2},[0.7,0.9],[0,0.1]\right\rangle,\left\langle x_{3},[0.4,0.6],[0.2,0.4]\right\rangle \\
& \left.\left\langle x_{4},[0.3,0.5],[0.2,0.4]\right\rangle\right\} \\
D_{4}^{\prime} & =\left\{\left\langle x_{1},[0.8,0.9],[0,0.1]\right\rangle,\right. \\
& \left\langle x_{2},[0.7,0.8],[0.1,0.2]\right\rangle,\left\langle x_{3},[0.7,0.9],[0,0.1]\right\rangle, \\
& \left.\left\langle x_{4},[0.8,0.9],[0,0.1]\right\rangle\right\}
\end{aligned}
$$

Suppose that the patient $P^{\prime}$ has all the symptoms, which is represented by the following interval-valued intuitionistic fuzzy set:

$$
\begin{aligned}
& P^{\prime}(\text { patient })=\left\{\left\langle x_{1},[0.4,0.5],[0.2,0.3]\right\rangle,\right. \\
& \quad\left\langle x_{2},[0.7,0.8],[0.1,0.2]\right\rangle,\left\langle x_{3},[0.9,1],[0,0.1]\right\rangle, \\
& \left.\quad\left\langle x_{4},[0.3,0.5],[0.2,0.4]\right\rangle\right\} .
\end{aligned}
$$

The weight vector of $\left(x_{1}, x_{2}, x_{3}, x_{4}\right)$ is $(0.25,0.4,0.15,0.2)$. Using the cosine similarity measure between IVIFSs in [22], we can calculate the interval-valued intuitionistic fuzzy cosine similarity measure $C_{\mathrm{IVIFS}}$, the interval-valued intuitionistic fuzzy weighted cosine similarity measure $C_{\text {IVIFWS }}$, and the interval-valued intuitionistic fuzzy ordered weighted cosine similarity measure $C_{\text {IVIFows }}$ between $P^{\prime}$ and $D_{i}^{\prime}(i=$ $1,2,3,4)$, respectively, and the results are shown in Table 8.

As we can see from Table 8 , the patient $P^{\prime}$ is assigned to the diagnosis $D_{2}^{\prime}$ (typhoid), and the result is the same as the method that we presented in this paper. According to the comparative analysis of Sections 4.1 and 4.2, the cosine similarity measures in this paper have the following advantage. It is reasonable that the proposed cosine similarity measures between IFLSs and IVIFLSs are defined based on linguistic scale function $f^{*}$, the decision-makers can flexibly select the linguistic scale function $f^{*}$ depending on 
their preferences and the actual semantic situations, and the decision-maker can employ it to address practical decisionmaking problems with precision.

\section{Conclusions}

In this paper, we propose the cosine similarity measures for IFLSs and IVIFLSs, which are expressed by the cosine function based on linguistic scale function. Then, the weighted cosine similarity measure and the ordered weighted cosine similarity measure for intuitionistic fuzzy linguistic term sets are introduced. The main advantage of the proposed cosine similarity measures is that the decision-makers can flexibly select the linguistic scale function depending on the actual semantic situation. Furthermore, we present the application of the cosine similarity measures for intuitionistic fuzzy linguistic term sets and interval-valued intuitionistic fuzzy linguistic term sets to pattern recognition and medical diagnosis, and the existing cosine similarity measures are compared with the proposed cosine similarity measures by the illustrative example. In future research, the developed cosine similarity measures will be extended to the intuitionistic fuzzy uncertain linguistic sets and it can be applied in other related decision-making.

\section{Conflicts of Interest}

The authors declare that they have no conflicts of interest.

\section{Acknowledgments}

This research is fully supported by a grant by National Natural Science Foundation of Hunan (2015JJ6041), by National Natural Science Foundation of China (11501191), by National Social Science Foundation of China (15BTJ028), by Major Projects of the National Social Science Foundation of China (17ZDA046), and by the Key International Collaboration Project of the National Nature Science Foundation of China (no. 71210003, Research on Electronic Business Based on the Users Behavior).

\section{References}

[1] L. A. Zadeh, "Fuzzy sets," Information and Control, vol. 8, no. 3, pp. 338-353, 1965.

[2] R. R. Yager, "Multiple objective decision-making using fuzzy sets," International Journal of Man-Machine Studies, vol. 9, no. 4, pp. 375-382, 1977.

[3] V. Khatibi and G. A. Montazer, "Intuitionistic fuzzy set vs. fuzzy set application in medical pattern recognition," Artificial Intelligence in Medicine, vol. 47, no. 1, pp. 43-52, 2009.

[4] S. Cateni, M. Vannucci, and V. Colla, "Industrial multiple criteria decision making problems handled by means of fuzzy inference-based decision support systems," in Proceedings of the 4th International Conference on Intelligent Systems, Modelling and Simulation, ISMS 2013, pp. 12-17, Thailand, January 2013.

[5] K. T. Atanassov, "Intuitionistic fuzzy sets," Fuzzy Sets and Systems, vol. 20, no. 1, pp. 87-96, 1986.

[6] F. Herrera and J. L. Verdegay, "Linguistic assessments in group decision," in Proceedings of the First European Congress on Fuzzy and Intelligent Technologies, pp. 941-948, Aachen, Germany, 1993.

[7] F. Herrera, E. Herrera-Viedma, and J. L. Verdegay, "A model of consensus in group decision making under linguistic assessments," Fuzzy Sets and Systems, vol. 78, no. 1, pp. 73-87, 1996.

[8] F. Herrera, E. Herrera-Viedma, and J. L. Verdegay, "Direct approach processes in group decision making using linguistic OWA operators," Fuzzy Sets and Systems, vol. 79, no. 2, pp. 175190,1996

[9] Z. S. Xu, "An approach based on the uncertain LOWG and induced uncertain LOWG operators to group decision making with uncertain multiplicative linguistic preference relations," Decision Support Systems, vol. 41, no. 2, pp. 488-499, 2006.

[10] Z. Xu, Uncertain Multi-Attribute Decision Making: Methods and Applications, Tsinghua University, 2015.

[11] X.-F. Wang, J.-Q. Wang, and W.-E. Yang, "Multi-criteria group decision making method based on intuitionistic linguistic aggregation operators," Journal of Intelligent \& Fuzzy Systems. Applications in Engineering and Technology, vol. 26, no. 1, pp. 115-125, 2014.

[12] H. Guan, S. Guan, and A. Zhao, "Intuitionistic fuzzy linguistic soft sets and their application in multi-attribute decisionmaking," Journal of Intelligent \& Fuzzy Systems: Applications in Engineering and Technology, vol. 31, no. 6, pp. 2869-2879, 2016.

[13] R. R. Yager, "Multi-criteria decision making with ordinal/linguistic intuitionistic fuzzy sets," IEEE Transactions on Fuzzy Systems, vol. 24, no. 3, pp. 590-599, 2015.

[14] Y. Pang and W. Yang, "Some hesitant intuitionistic fuzzy linguistic distance measures," International Journal of Innovative Computing, Information and Control, vol. 11, no. 5, pp. 15731586, 2015.

[15] W. Yang, Y. Pang, J. Shi, and C. Wang, "Linguistic hesitant intuitionistic fuzzy decision-making method based on VIKOR," Neural Computing and Applications, pp. 1-14, 2016.

[16] S. Santini and R. Jain, "Similarity measures," IEEE Transactions on Pattern Analysis and Machine Intelligence, vol. 21, no. 9, pp. 871-883, 1999.

[17] S. M. Mulekar and C. S. Brown, "Distance and similarity measures," in Encyclopedia of Social Network Analysis and Mining, pp. 385-400, Springer, New York, NY, USA, 2015.

[18] J. Ye and Q. Zhang, "Single valued neutrosophic similarity measures for multiple attribute decision-making," Neutrosophic Sets and Systems, 2015.

[19] S.-H. Cheng, S.-M. Chen, and W.-S. Jian, "Fuzzy time series forecasting based on fuzzy logical relationships and similarity measures," Information Sciences, vol. 327, pp. 272-287, 2016.

[20] J. Ye, "Cosine similarity measures for intuitionistic fuzzy sets and their applications," Mathematical and Computer Modelling, vol. 53, no. 1-2, pp. 91-97, 2011.

[21] L. Zhou, Z. Tao, H. Chen, and J. Liu, "Intuitionistic fuzzy ordered weighted cosine similarity measure," Group Decision \& Negotiation, vol. 23, no. 4, pp. 879-900, 2014.

[22] D. Liu, X. Chen, and D. Peng, "Interval-valued intuitionistic fuzzy ordered weighted cosine similarity measure and its application in investment decision-making," Complexity, vol. 2017, Article ID 1891923, 11 pages, 2017.

[23] J.-Q. Wang, J.-T. Wu, J. Wang, H.-Y. Zhang, and X.-H. Chen, "Multi-criteria decision-making methods based on the Hausdorff distance of hesitant fuzzy linguistic numbers," Soft Computing, vol. 20, no. 4, pp. 1621-1633, 2016. 
[24] R. R. Yager, "On ordered weighted averaging aggregation operators in multicriteria decisionmaking," IEEE Transactions on Systems, Man, and Cybernetics, vol. 18, no. 1, pp. 183-190, 1988.

[25] G. Salton and M. J. Mcgill, Introduction to Modern Information Retrieval, McGraw-Hill, 1983.

[26] K. Atanassov and G. Gargov, "Interval valued intuitionistic fuzzy sets," Fuzzy Sets and Systems, vol. 31, no. 3, pp. 343-349, 1989.

[27] K. T. Atanassov, Intuitionistic Fuzzy Sets, Physica-Verlag, New York, NY, USA, 1999. 


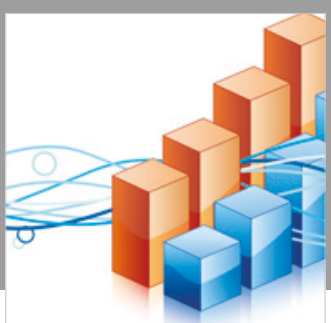

Advances in

Operations Research

\section{-n-m}
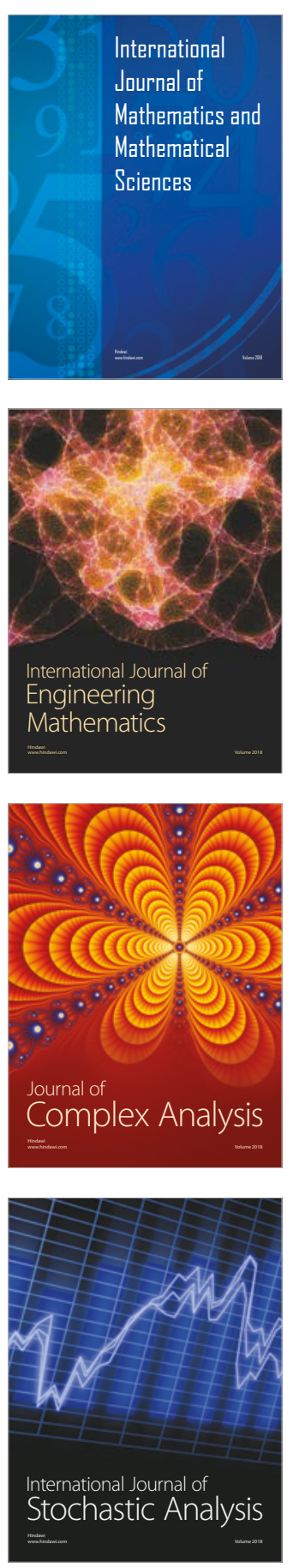
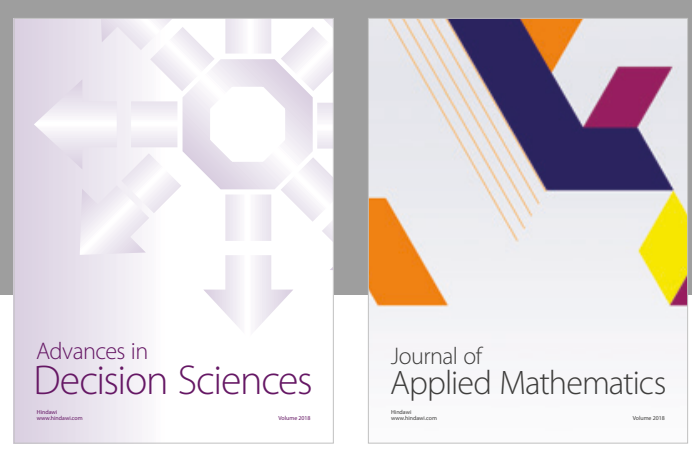

Journal of

Applied Mathematics
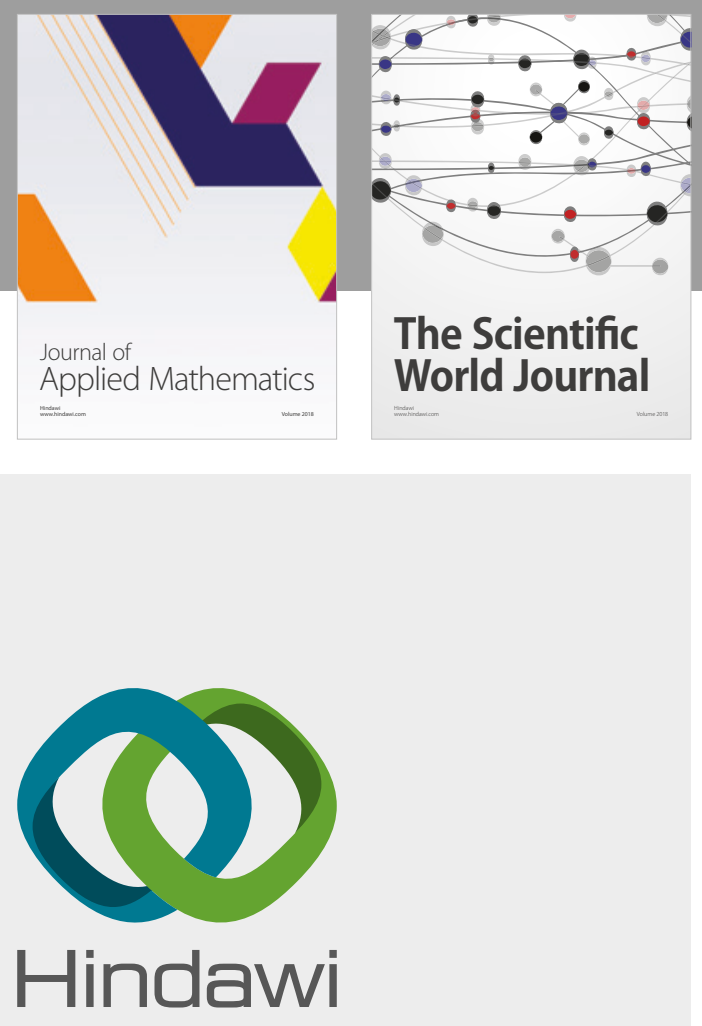

Submit your manuscripts at

www.hindawi.com

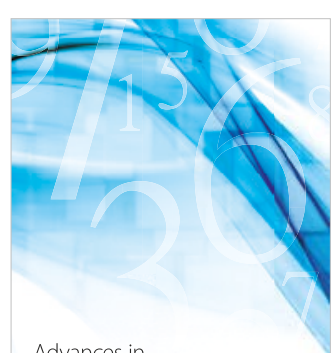

Advances in
Numerical Analysis
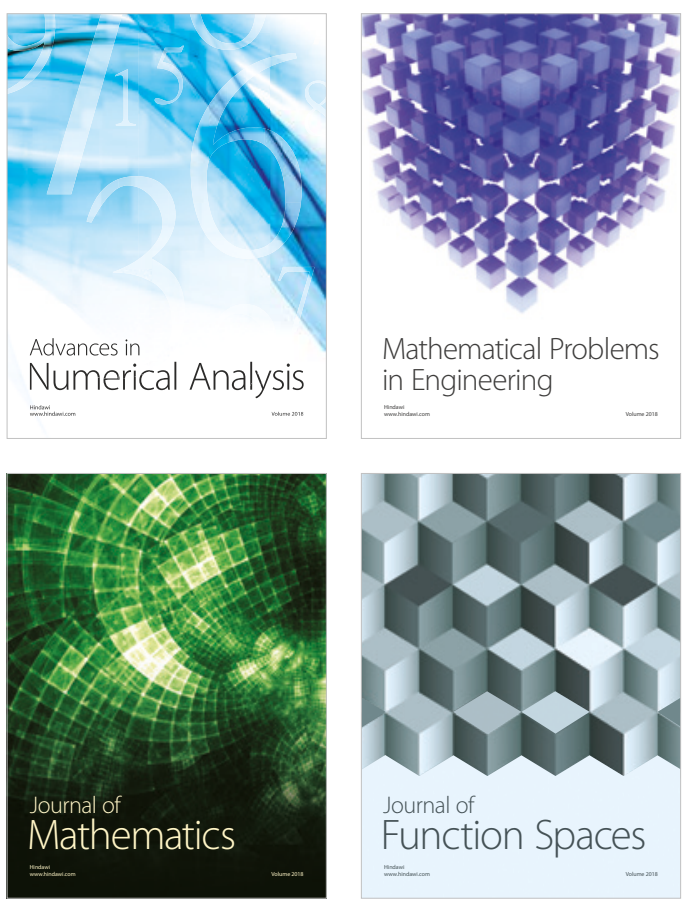

Mathematical Problems in Engineering

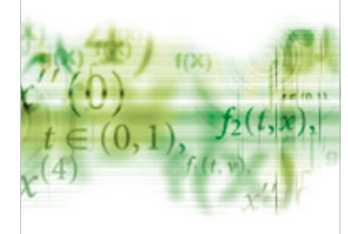

International Journal of

Differential Equations

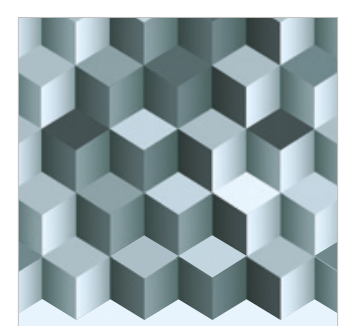

Journal of

Function Spaces
The Scientific

World Journal

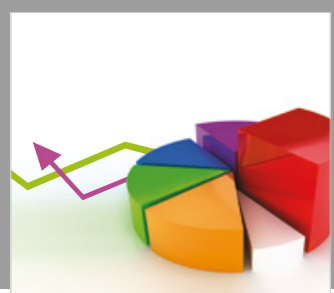

Journal of

Probability and Statistics
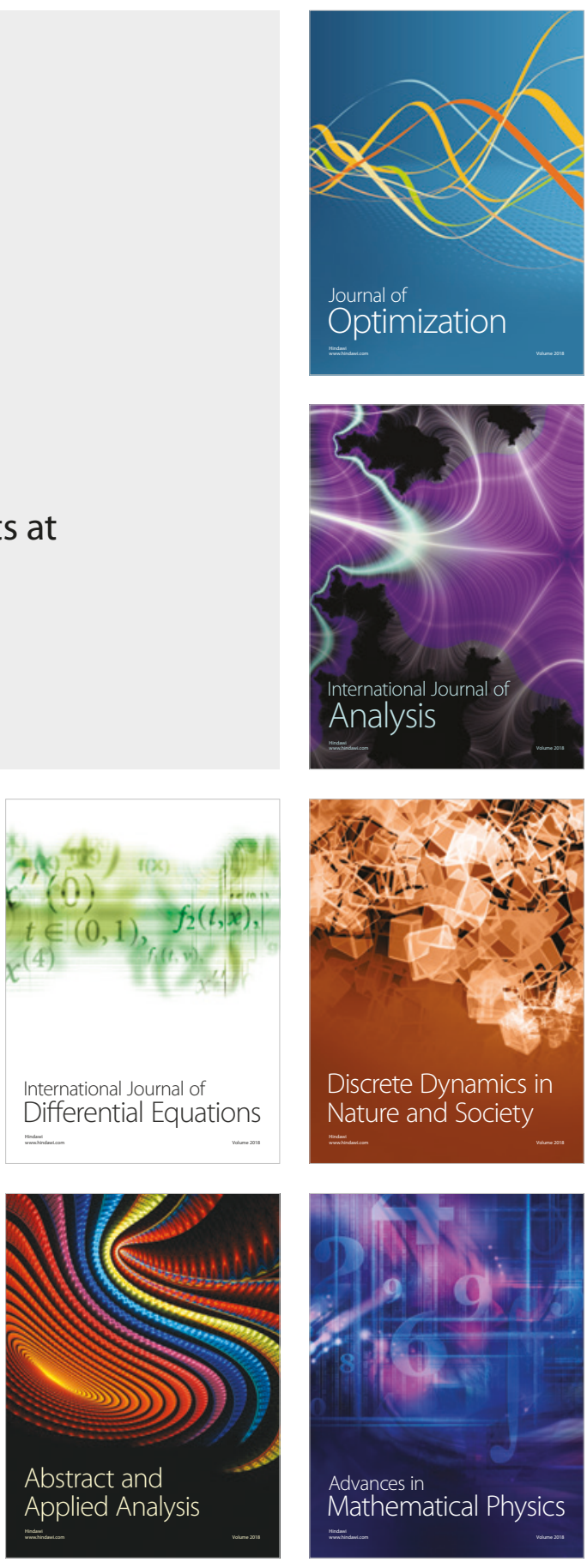